\title{
On the transition from localized to delocalized electronic states in divalent-metal clusters
}

\author{
M.E. Garcia ${ }^{1}$, G.M. Pastor ${ }^{2}$, and K.H. Bennemann ${ }^{1}$ \\ 1 Institut für Theoretische Physik der Freien Universität Berlin, Arnimallee 14, W-1000 Berlin 33, Federal Republic of Germany \\ 2 Institut für Theoretische Physik der Universität zu Köln, Zülpicher Strasse 77, W-5000 Köln 41, Federal Republic of Germany
}

Received 10 September 1990

\begin{abstract}
The transition from van der Waals to covalent bonding, which is expected to occur in divalent-metal clusters with increasing cluster size, is discussed. We propose a model which takes into account, within the same electronic theory, the three main competing contributions, namely the kinetic energy of the electrons, the Coulomb interactions between electrons, and the $s \leftrightarrow p$ intraatomic transitions responsible for van der Waals like bonding. The model is solved by taking into account electron correlations using a generalized Gutzwiller approximation (slave boson method). The occurrence of electron localization is studied as a function of the interaction parameters and cluster size.
\end{abstract}

PACS: $36.40 .+\mathrm{d}$

The evolution of the electronic properties from the atom to the bulk is one of the main problems of cluster physics. An important magnitude in this respect is the electronic kinetic energy of a cluster, whose deviation from the atomic kinetic energy as a function of the cluster size must change entirely if, for example, the bulk shows semiconducting or metallic behaviour. This is the case for the alkali-metal clusters, in which the valence electrons become itinerant already for small clusters [1]. The situation should be different for divalent metal clusters, where the closed-shell atomic configuration might cause in the small clusters a rather atomic like behaviour. For instance, the $\mathrm{Hg}$ atom has a $[\mathrm{Xe}] 4 f^{14} 5 d^{10} 6 s^{2}$ electronic configuration, a considerably large $6 s^{2} \rightarrow 6 s 6 p$ transition energy of $\sim 6 \mathrm{eV}$, and a high atomic polarizability. Consequently, van der Waals type interactions arising from intraatomic $s \leftrightarrow p$ transitions are expected to dominate for small cluster sizes, causing the valence electrons to be mainly localized in atomic like states. Since the $\mathrm{Hg}$ bulk is a metal, one expects a drastic change in the itinerant character of the electrons as $n$ increases.

Recently, experiments and calculations [2-6] have been performed on $\mathrm{Hg}_{n}$ clusters in order to characterize the transition from atomic-like to bulk-like behaviour. Comparison between experiment and theory suggests $[5,6]$ that the nature of the chemical bonding as a function of $n$ can be divided into three different regimes, namely van der Waals bonding for $n \leqslant 13-19$, covalent for $13-19 \lesssim n \lesssim 80$, and metallic behaviour for $n \gtrsim 80$. However, previous Tight-binding calculations of the ionization potential $[5,6]$ and of the $5 d \leftrightarrow 6 p$ autoionization energy of $\mathrm{Hg}_{n}$ clusters yield a rather smooth transition in contrast to the experimental results. The agreement with experiment could be improved assuming that the electrons occupy atomic like states (the hopping elements are set to be zero) for $n \lesssim 13$ in the case of the ionization potential, and for $n \lesssim 20$ for the autoionization energy. Evidently, previous calculations can only account for the transition by proposing for the small clusters localized electrons, and for larger clusters delocalized electrons. An electronic theory, which includes both van der Waals and covalent bonding and their interplay as a function of the cluster size is necessary. Note, the same valence electrons are involved in both types of bonding. Such a theory should be able to reproduce the trends observed for the other divalent metals, and to determine which type of bonding dominates for a given element, and a given cluster size.

Thus, we develop a model, which for the first time takes into account, on the same footing, the fundamental interplay between kinetic and Coulomb energies (of Hubbard type) and the van der Waals interactions (resulting from the coupling between $s \leftrightarrow p$ and local excitations, induced-dipole-induced-dipole-interactions) [10].

The ground state properties of $\mathrm{Hg}_{n}$ are calculated by using a generalization [7] of the slave bosons method in the saddle point approximation [8], which has been proved to be equivalent to the Gutzwiller [9] approach. We consider the following local electronic configurations, which are expected to contribute dominantly to the true ground state:

$\left|\psi_{i s^{2}}\right\rangle \equiv c_{i s \uparrow}^{+} c_{i s \downarrow}^{+}|0\rangle$,

$\left|\psi_{i s \sigma}\right\rangle \equiv c_{i s \sigma}^{+}|0\rangle$, 
$\left|\psi_{i s^{2} p \sigma}\right\rangle \equiv c_{i s \uparrow}^{+} c_{i s \downarrow}^{+} c_{i p \sigma}^{+}|0\rangle$,

$\left|\psi_{\text {isp } \sigma \bar{\sigma}}\right\rangle \equiv c_{i s \sigma}^{+} c_{i p \bar{\sigma}}^{+}|0\rangle$.

$\left|\psi_{i s^{2}}\right\rangle$ refers to the doubly occupied $6 s^{2}$ atomic configuration, $\left|\psi_{i s \sigma}\right\rangle$ to the singly occupied $6 s$ states, $\left|\psi_{i s^{2} p \sigma}\right\rangle$ to the triply occupied $6 s^{2} 6 p$ configurations, and $\left|\psi_{i s p o \sigma}\right\rangle$ to the mixed $6 s 6 p$ states, in which the $s$ and $p$ electrons have opposite spins (s. Fig. 1). We put aside all other possible electronic configurations at site $i$, i.e., we will construct the ground state only with the states given in (1). This is a reasonable assumption if $\Delta=\varepsilon_{p}-\varepsilon_{s}$ and the intraatomic Coulomb integrals $U_{s s}$ and $U_{s p}$ are large, which is the case for $\mathrm{Hg}_{n}$. For simplicity, the $p$ orbitals are taken to be $s$-like, so we do not distinguish between $p_{x}, p_{y}$ and $p_{z}$ orbitals.

The states $\left|\psi_{i s \sigma}\right\rangle$ and $\left|\psi_{i s^{2} p \sigma}\right\rangle$ describe the charge fluctuations, and the weights of these configurations in the ground state give a measure of the strength of the covalent character and the itinerancy of the electrons in the cluster; the states $\left|\psi_{\text {ispo } \sigma}\right\rangle$ represent the local $s \leftrightarrow p$ excitations, and are expected to contribute appreciably to the ground state only if van der Waals interactions are present.

Following Kotliar and Ruckenstein [8] we enlarge the Fock space by including a set of bosons, which correspond to the configurations defined in (1) (Fig. 1). The boson creation (annihilation) operators $d_{i}^{+}\left(d_{i}\right)$ (s doubly occupied), $s_{i}^{+}\left(s_{i}\right)$ (s singly occupied), $t_{i}^{+}\left(t_{i}\right)$ ( $s^{2} p$ triply occupied) and $m_{i \sigma \sigma}^{+}\left(m_{i \sigma \sigma}\right)$ (mixed $\left.s p\right)$ act as projection operators upon the states $\left|\psi_{i s^{2}}\right\rangle,\left|\psi_{i s \sigma}\right\rangle,\left|\psi_{i s^{2} p \sigma}\right\rangle$ and $\left|\psi_{i s p \sigma \sigma}\right\rangle$ respectively.

In the saddle-point approach, the boson operators are replaced by numbers to be determined from minimizing the energy of the ground state. This is equivalent to assume that the quantum fluctuations are negligible. Then, the energy per atom (for the paramagnetic state) can be written as

$E(n)=E_{\mathrm{band}}+E_{\mathrm{Cou}:}+E_{\mathrm{vdW}}$.

The first term of (2) corresponds to the kinetic energy of the electrons $E_{\text {band }}=2 \int \mathrm{d} \varepsilon\left(\varepsilon-\tilde{\varepsilon}_{s}\right) \tilde{N}_{s \sigma}(\varepsilon)+2 \int \mathrm{d} \varepsilon\left(\varepsilon-\tilde{\varepsilon}_{p}\right)$ $\tilde{N}_{p \sigma}(\varepsilon)$, where $\tilde{N}_{s}\left(N_{p}\right)$ are the local $s(p)$ density of states resulting from an effective tight-binding Hamiltonian with

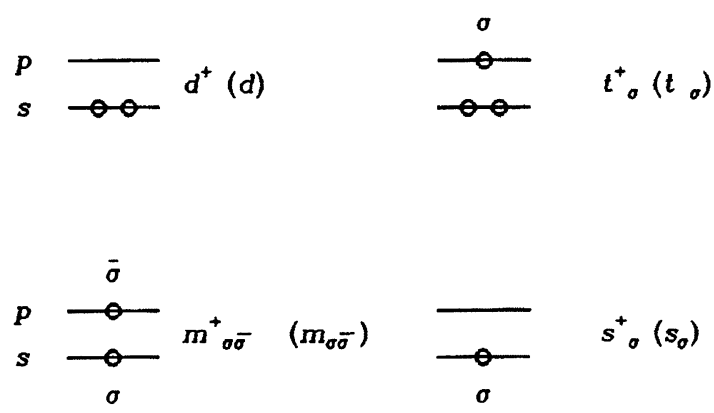

Fig. 1. Illustration of the configurations taken into account in the slave-bosons calculation. $d^{+}(d)$ refer to the creation (annihilation) operators of the doubly occupied $s$ state, $m_{\sigma \sigma}^{+}\left(m_{\sigma \sigma}\right)$ to the mixed $s p$ configuration, $t_{\sigma}^{+}\left(t_{\sigma}\right)$ to the triple $s^{2} p$ states, and $s_{\sigma}^{+}\left(s_{\sigma}\right)$ to the single $s$ electron configuration parameters $\tilde{\varepsilon}_{s}, \tilde{\varepsilon}_{p}, \tilde{t}_{s s}, \tilde{t}_{s p}, \tilde{t}_{p p p}$ which depend on $d^{2}, m^{2}, s^{2}$ and $t^{2} . \quad E_{\text {Coul }}=U_{s s}\left(d^{2}+2 t^{2}-1\right)+2 U_{s p}\left(2 t^{2}+m^{2}\right)$ describes the Coulomb energy of the charge fluctuations arising from the jumps of $s$ electrons to the $p$ level of neighbouring atoms. $U_{s s}$ and $U_{s p}$ are the intraatomic coulomb integrals. $E_{v d W}=V^{2} z \frac{\left(q^{v}\right)^{2}}{\left(\tilde{\varepsilon}_{p}-\tilde{\varepsilon}_{s}\right)}$ refers to the van der Waals energy. $z$ refers to the coordination number, $V$ to the strength of the interaction and $q^{v}$ its corresponding renormalization factor, which depends mainly on $d^{2}$ and $m^{2}$ (i.e., on the configurations involved in the local $s \leftrightarrow p$ excitations which give rise to the van der Waals attraction $[7,10])$.

The energy $E(n)$ is minimized with respect to the variables $d, s, t$ and $m$, taking into account that these variables are related by the following constraints

$2 t^{2}+2 s^{2}+2 m^{2}+d^{2}=1$,

$n_{p} / 2=m^{2}+t^{2}$

$n_{s} / 2=2 t^{2}+s^{2}+d^{2}+m^{2}$.

The completeness relation of (3a) reflects the fact that the probability for the system to be in one of the considered states must be one. Eqs. (3b) indicate the way of counting the number of $s$ and $p$ electrons in terms of the probabilities for the different local configurations. Since $\Delta$ and $U_{s p}$ are rather large in $\mathrm{Hg}\left(U_{s p} \simeq 6.5 \mathrm{eV}\right.$ [5] we do not expect a strong redistribution of charge within the neutral cluster. Therefore, we set $n_{s}+n_{p}=2$ for all cluster sites. Thus, from (3a)-(3b) $s^{2}=t^{2}$, which indicates that single and triple states involved in charge fluctuations are equally probable. This is, however, related to our choice of the states which contribute to the ground state (see (1)). The renormalization of the hopping elements is given by

$q^{t}=\frac{t^{2}(d+m)^{2}}{\left(m^{2}+t^{2}\right)\left(m^{2}+3 t^{2}+d^{2}\right)}$,

From (2) and (4) it is possible to analyze the effect of the Coulomb and van der Waals interactions on the kinetic energy of the electrons. For $U_{s p} \rightarrow \infty$ charge fluctuations become energetically unfavourable, and consequently $t^{2} \rightarrow 0$ in order to minimize $E(n)$. If van der Waals interactions are not present, one expects $m^{2} \rightarrow 0$, since the mixed $s p$ state is not directly connected with the $s^{2}$ configuration. Thus, from $(3,4)$ results $q^{t} \rightarrow 1$. Charge fluctuations are almost suppressed by the large $U_{s p}$ but the electrons are still delocalized in order to gain kinetic energy and give rise to a nonzero cohesive energy. However, a completely different situation occurs if $V \neq 0$. As soon as van der Waals interactions are turned on, $m^{2} \neq 0$, and $q^{x} \rightarrow 0$ for $t^{2} \rightarrow 0$, i.e., the kinetic energy of the electrons is atomic like, the electrons remain localized and take part in an alternative mechanism of cohesion, which does not involve charge fluctuations. The competition between both types of bonding can be also clearly seen from the completeness relation (3a), since an increase of $m^{2}$ implies a decrease of $t^{2}$.

We have minimized the free energy (2) with respect to the probabilities for the configurations of Fig. 1. If $V \neq 0$ we find, for a fixed cluster size, two minima, one for $q^{t}=1$ 


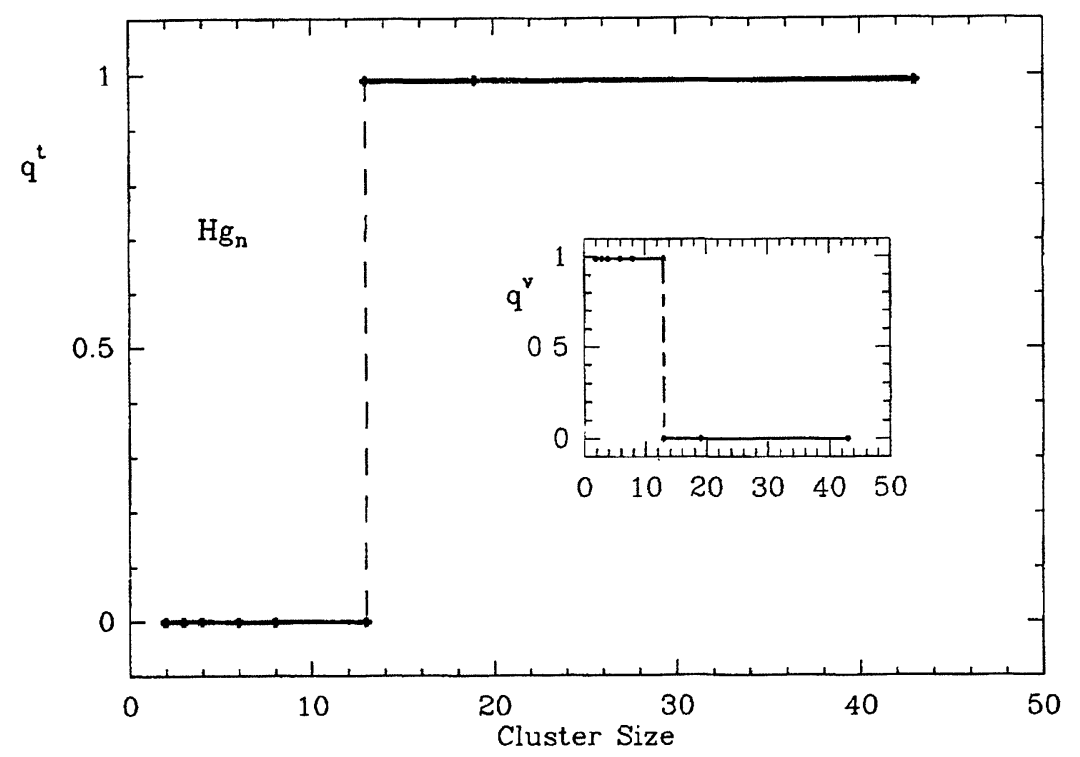

Fig. 2. Renormalized hopping element $q^{l}$ as a function of the cluster size. In the inset figure results for the renormalized van der Waals coupling constant $q^{v}$ are given. For $n \geq 19$ the electrons delocalize and form covalent bonds $\left(q^{t}=1\right)$, whereas van der Waals is completely suppressed $\left(q^{v}=0\right)$. The inverse situation holds for $n \leq 8$ and $q^{v}=0$ (covalent minimum), and another one for $q^{t}=0$ and $q^{v}=1$ (van der Waals minimum). This indicates that the cluster is either covalent or van der Waals bounded. For increasing $U_{s p}=U_{s s}$ the covalent solution $\left(E\left(q^{t}=1\right)\right)$ decreases, whereas van der Waals $\left(E\left(q^{t}=0\right)\right)$ remains unaltered. The decrease of the covalent minimum for large $U_{s p}$ is in good agreement with the energy calculated using second order perturbation theory on the state $\left|\psi_{i s^{2}}\right\rangle$. We find a crossing of the van der Waals and covalent solutions at a critical value $\left(U_{c r}\right)$ of $U_{s p} .\left(U_{c r}(n)\right)$ increases rather slowly with increasing cluster size. This implies that a transition from localized to delocalized electronic states occurs as a function of $n$.

The parameters used for $\mathrm{Hg}$ are $\Delta=5.8 \mathrm{eV}$, tss $=$ $-0.6 \mathrm{eV}, t s p=-0.6 \mathrm{eV}, t p p=-1.0 \mathrm{eV}, U_{s s}=U_{s p}=$ $6.5 \mathrm{eV}$ and $V=0.6 \mathrm{eV}$. One gets the transition from van der Waals to covalent bonding between $n=8$ and $n=19$. The value of $n_{c r}$ is very sensitive to the value of the Coulomb integrals, i.e., small changes in $U_{s p}$ can shift the transition considerably. This can be understood as follows. The van der Waals energy increases approximately proportional to the number of bonds. The energy of a covalent bond can be approximated, for small cluster sizes, by $4 t_{s p}^{2} /\left(\Delta+U_{s p}\right)$. Hence, the covalent energy per bond remains also approximately constant. If both van der Waals and covalent energies per bond were constant, no transition would take place as a function of the cluster size. We conclude that, as physically expected, the screening has to be taken into account in order to account properly for the transition. Charge fluctuations are screened better in large clusters. Consequently the covalent energy per bond increases with respect to the smaller clusters and can overcome the van der Waals energy [7].

At $n_{c r} \simeq 8-19$ there is a sharp transition, where $q^{t}$ increases from 0 to 1 and $q^{v}$ decreases from 1 to 0 , as shown in Fig. 2. Although such a sharp transition could be inferred from some experiments [2-4], one should note that the mutual exclusion of covalent and van der Waals bonding mechanisms might be exaggerated by our saddle point approach. The correlations taken into account in our model seem to describe the essential aspects of the competition between kinetic, Coulomb and van der Waals energies, but the quantum fluctuations, which we have disregarded in our saddle point scheme, can play an important role for small clusters. We feel that the most realistic physical picture for divalent metal clusters is a coexistence between both types of bonding. The van der Waals bonding dominates for small clusters, while the covalent bonding is more important for larger clusters. Exact results for the dimer support this [7].

This work was supported by the Deutsche Forschungsgemeinschaft, SFB 337 and SFB 341.

\section{References}

1. Koutecký, J., Fantucci, P.: Chem. Rev. 86, 539 (1986)

2. Rademann, K., Kaiser, B., Even, U., Hensel, F.: Phys. Rev. Lett. 59, 2319 (1987)

3. Breéchignac, C., Broyer, M., Cahuzac, Ph., Delacretaz, G., Labastie, P., Wolf, J.P., Wöste, L.: Phys. Rev. Lett. 60, 275 (1988)

4. Haberland, H., Kornmeier, H., Langosch, H., Oschwald, M., Tanner, G.: J. Chem. Soc. Faraday Trans. 86, 1 (1990)

5. Pastor, G.M.: Thesis, Freie Universität Berlin, 1989

6. Pastor, G.M., Stampfli, P., Bennemann, K.H.: Phys. Sci. 38, 623 (1988); Europhys. Lett. 7, 419 (1988)

7. Garcia, M.E., Pastor, G.M., Bennemann, K.H.: (to be published)

8. Kotliar, G., Ruckenstein, A.E.: Phys. Rev. Lett. 57, 1362 (1986)

9. Gutzwiller, M.: Phys. Rev. Lett. 10, 159 (1963)

10. Langbein, D.: Theory of Van der Waals attraction. In: Springer Tracts in Modern Physics. Vol. 72. Berlin, Heidelberg, New York: Springer 1974 